\title{
OMISSÃO DE MACRONUTRIENTES EM PLANTAS DE AMENDOIM ( $\left.{ }^{1}\right)$
}

\author{
FRANCISCO SOLANO DE OLIVEIRA RODRIGUES FILHO $\left({ }^{2}\right)$, \\ CELI TEIXEIRA FEITOSA $\left({ }^{3,4}\right)$ e MARCELO APARECIDO NUNES GERIN $\left({ }^{2}\right)$
}

\begin{abstract}
RESUMO
Plantas de amendoim Arachis hypogaea L. Tatu' foram cultivadas em condiçōes de casa de vegetação, em vasos de Mitscherlich contendo areia lavada e irrigados com solução nutritiva completa e com soluçōes nutritivas com a omissāo de cada macronutriente ( $\mathrm{N}, \mathrm{P}, \mathrm{K}, \mathrm{Ca}, \mathrm{Mg}$ e S). As plantas mostraram sintomas de deficiência de macronutrientes, com exceção do fơsforo, na ausência de cada elemento, relacionados com os seus baixos teores e redução no crescimento, desenvolvimento e produção de matéria seca.
\end{abstract}

Termos de indexação: amendoim, deficiência de macronutrientes.

(1) Entregue para publicação em 25 de fevereiro de 1988 e aceito em 30 de junho de 1988.

(2) Seção de Oleaginosas, Instituto Agronómico (IAC), Caixa Postal 28, 13001 Campinas (SP).

(3) Seção de Fertilidade do Solo e Nutrição de Plantas, IAC.

(4) Com bolsa de pesquisa do CNPq. 


\section{INTRODUÇÃO}

Entre os objetivos do estudo de nutrição mineral das plantas estão a caracterização das deficiências dos elementos nutritivos e as conseqüências no desenvolvimento e composição das plantas. O sistema biológico, no caso da planta, deve ser considerado em termos de equilíbrio entre os ions e seus complexos, pois os processos fisiológicos e bioquímicos de desenvolvimento das plantas sāo controlados, em parte, pelo fomecimento dos elementos nutritivos. Assim, planta e solo devem estar nutricionalmente compativeis, de modo a permitir, com maior eficiência, o máximo de aproveitamento pela planta e o máximo de fornecimento pelo solo dos elementos nutritivos e, conseqüentemente, a obtenção de plantas bem nutritivas e vigorosas que possam oferecer produções elevadas.

No caso específico da cultura do amendoim, pouco tem sido realizado, no Brasil, no tocante à nutrição mineral e em particular com respeito a sintomas de deficiência e toxicidade de nutrientes. Além disso, a informação disponivel sobre o efeito de deficiências minerais em amendoim não é completa. Alguns trabalhos, como o de HARRIS \& BLEDSOE (1951) e REID \& YORK JR. (1958),caracterizaram os sintomas de deficiência de elementos minerais em amendoim. Esses trabalhos, porém, foram feitos com variedades nāo utilizadas em nossas condições e seguindo um método diferente do nosso, em vários aspectos.

O presente trabalho visou caracterizar os sintomas de deficiência de macronutrientes e estudar o efeito da omissão de $\mathrm{N}, \mathrm{P}, \mathrm{K}, \mathrm{Ca}, \mathrm{Mg}$ e $\mathrm{S}$ sobre a composição quimica, o peso de matéria seca e o desenvolvimento de plantas de amendoim.

\section{MATERIAL E MÉTODOS}

Sementes de amendoim 'Tatu' foram colocadas para germinar e crescer, por trinta dias, em vasos contendo areia lavada e irrigados com soluçāo nutritiva completa e soluções nutritivas com omissão de cada um dos macronutrientes. A solução nutritiva completa empregada foi a de HOAGLAND \& ARNON (1950), modificada por SARRUGE \& HAAG (1975). Para os casos de omissão, a solução nutritiva foi elaborada sem a adição do macronutriente correspondente. No tratamento sem $\mathrm{N}$, aplicaram-se no começo da germinação $5 \mathrm{mg}$ de $\mathrm{N}$ por vaso na forma de nitrato de amônio para o arranque inicial da cultura.

$O$ delineamento experimental foi de blocos ao acaso, com sete tratamentos e cinco repetiçōes, a saber: 1. Completo; 2 . Sem N; 3. Sem P; 4. Sem K; 5. Sem Ca; 6. Sem Mg, e 7. Sem S. 
Lavou-se com $\mathrm{HCl}$ a areia de praia utilizada como substrato, várias vezes, para descontaminação, analisando-a, a seguir, para detecção de possiveis resíduos minerais.

No plantio com sementes do cultivar 'Tatu', empregaram-se seis sementes por vaso, deixando-se, após a germinação, apenas duas plantas por vaso. Os vasos do tipo Mitscherlich, com capacidade para $5 \mathrm{~kg}$, contendo areia lavada, foram irrigados com as soluções nutritivas até o final do experimento, aos trinta dias do plantio, quando as plantas foram fotografadas, anotando-se as alturas e os sintomas de deficiências nutricionais. Em seguida, colheu-se a parte aérea, que foi lavada com água destilada e seca a $60^{\circ} \mathrm{C}$, até peso constante, obtendo-se o peso seco total da parte aérea das plantas. Após a pesagem, o material foi moído, determinando-se os macronutrientes ( $\mathrm{N}, \mathrm{P}, \mathrm{K}, \mathrm{Ca}, \mathrm{Mg}$ e $\mathrm{S}$ ) e os micronutrientes ( $\mathrm{Fe}, \mathrm{Mn}, \mathrm{Cu}, \mathrm{Zn}, \mathrm{B}$ e Al), pelos métodos descritos por BATAGLIA et al. (1983).

\section{RESULTADOS E DISCUSSÃO}

No quadro 1 encontram-se os dados referentes ao peso da matéria seca da parte aérea e a altura das plantas.

QUADRO 1. Resultados de peso de matéria seca e de altura de plantas de amendoim, cultivadas em solução nutritiva completa e com a omissão de cada macronutriente, aos trinta dias de idade

\begin{tabular}{lrr}
\hline & g/vaso & $\mathrm{cm}$ \\
& & \\
Completo & $40,81 \mathrm{a}$ & $23,9 \mathrm{a}$ \\
Sem N & $8,80 \mathrm{c}$ & $4,5 \mathrm{~d}$ \\
Sem P & $23,02 \mathrm{~b}$ & $15,5 \mathrm{~b}$ \\
Sem K & $23,30 \mathrm{~b}$ & $13,6 \mathrm{bc}$ \\
Sem Ca & $7,10 \mathrm{c}$ & $6,0 \mathrm{~d}$ \\
Sem Mg & $9,20 \mathrm{c}$ & $6,5 \mathrm{~d}$ \\
Sem S & $14,43 \mathrm{bc}$ & $10,2 \mathrm{c}$ \\
\hline Tukey $5 \%$ & 10,62 & 3,65 \\
CV\% & 36,6 & 18,1 \\
\hline
\end{tabular}




\subsection{Peso da matéria seca}

Os resultados de peso da matéria seca, em gramas por vaso, mostraram, através da análise da variância, que houve efeito altamente significativo para tratamentos. Aqueles com a omissão de fósforo e potássio foram os que apresentaram as menores reduções do peso da matéria seca, em relação ao tratamento completo, com $43 \%$ para ambos os macronutrientes. Já os tratamentos com a omissão de cálcio, nitrogênio, magnésio e enxofre, registraram as maiores reduções de peso da matéria seca: $82,78,77$ e $65 \%$ respectivamente.

\subsection{Altura das plantas}

As medições de altura de planta mostraram diferença significativa entre os tratamentos. A menor redução de planta, em relação ao tratamento completo, foi obtida no tratamento com omissão de fósforo, $35 \%$. Os tratamentos com omissão de potássio, enxofre, magnésio, cálcio e nitrogênio apresentaram, respectivamente, $43,57,73,75$ e $81 \%$ de redução na altura da planta.

\subsection{Composição dos elementos nas plantas}

Pelo quadro 2 - composição química dos macronutrientes $(\mathrm{N}, \mathrm{P}, \mathrm{K}, \mathrm{Ca}$, $\mathrm{Mg}$ e S) na parte aérea - pode-se observar que o tratamento completo foi o que apresentou teor mais elevado de potássio, que variou de 3,58\%, para o tratamento completo, a $0,36 \%$, para o tratamento com omissão de potássio. $O$ mesmo não ocorreu com relação aos demais macronutrientes ( $N, P, \mathrm{Ca}, \mathrm{Mg}$ e S).

A omissão de potássio acarretou aumentos na absorção de $\mathrm{N}, \mathrm{P}, \mathrm{Ca}$, $\mathrm{Mg}$ e S, e a omissão de Ca levou a aumentos no teor de $\mathrm{N}$ e S.

O teor de nitrogênio, no tratamento completo, foi semelhante ao do tratamento com omissão de nitrogênio. Isso ocorreu, provavelmente, devido a uma aplicação de pequena quantidade de nitrogênio necessária para o arranque inicial das plantas adicionadas ao tratamento com a omissão do elemento: num teste preliminar à instalação e condução do presente trabalho, no tratamento com a omissão completa de nitrogênio, as plântulas não conseguiram desenvolver-se após a germinação das sementes, morrendo logo em seguida.

A omissão de nitrogênio acarretou diminuição no teor de magnésio e, em menor grau, no de potássio, enquanto a omissão de fósforo e de enxofre não ocasionou alteraçōes marcantes no teor dos macronutrientes em estudo. A omissão de cálcio acarretou aumento nos teores de nitrogênio e enxofre na parte aérea do amendoim. A omissão de magnésio mostrou efeito depressivo na ábsorção de fósforo, potássio e cálcio. 
QUADRO 2. Resultados dos teores de macronutrientes na parte aérea de plantas de amendoim cultivadas em solução nutritiva completa e com a omissão de cada macronutriente, aos trinta dias de idade

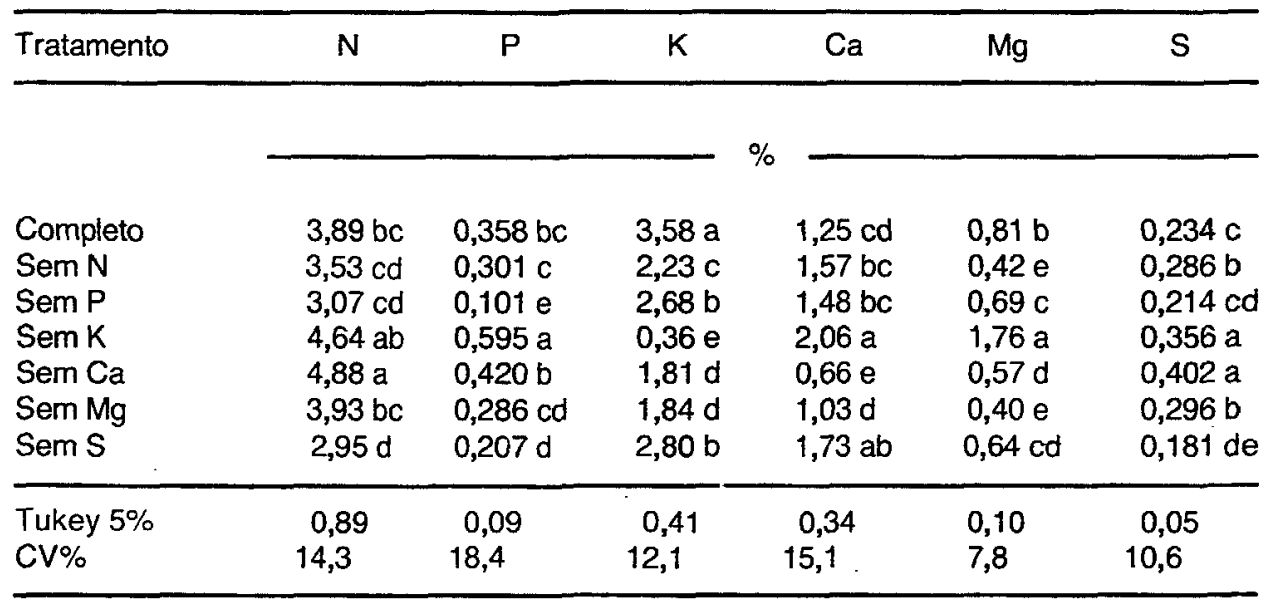

Com relação aos teores de micronutrientes, apesar de todos serem adicionados em doses iguais em todos os tratamentos, as omissōes dos macronutrientes contribuíram para que houvesse diferença em sua absorção (Quadro 3). Observaram-se aumentos mais acentuados quando se suprimiu o cálcio da solução nutritiva. $O$ boro foi o micronutriente que menor influência sofreu, tendo aumentado significativamente seu teor somente na ausência de fósforo e potássio. O Al foi bastante afetado pela omissão dos macronutrientes, ocorrendo, de modo geral, teores mais elevados do que o tratamento completo.

\subsection{Sintomas de deficiência}

A falta de qualquer elemento essencial à planta afeta seu crescimento, desenvolvimento e produção.

Sintomas de deficiências minerais em amendoim, raramente encontradas em condiçōes de campo, podem ser observados em plantas conduzidas em solução nutritiva (Figura 1).

A planta de amendoim cultivada em meio provido de quantidades adequadas de nitrogênio, fósforo, potássio, cálcio, magnésio, enxofre e micronutrientes, torna-se geralmente verde-escura, cresce rapidamente e produz grande quantidade de massa verde. 
QUADRO 3. Teores de micronutrientes na parte aérea de plantas de amendoim cultivadas em solução nutritiva completa e com a omissão de cada macronutriente, aos trinta dias de idade

\begin{tabular}{|c|c|c|c|c|c|c|}
\hline Tratamento & $\mathrm{Fe}$ & $\mathrm{Mn}$ & $\mathrm{Cu}$ & $\mathrm{Zn}$ & $B$ & $\mathrm{Al}$ \\
\hline & & & & $\%$ & & \\
\hline Completo & $89,6 \mathrm{c}$ & $49,8 \mathrm{c}$ & $8,2 \mathrm{c}$ & $32,0 \mathrm{c}$ & $55,6 \mathrm{c}$ & $69,0 \mathrm{~b}$ \\
\hline Sem N & $159,2 a b$ & $116,6 \mathrm{ab}$ & $21,5 \mathrm{a}$ & $73,7 \mathrm{c}$ & $65,6 \mathrm{bc}$ & $145,8 \mathrm{a}$ \\
\hline Sem P & $126,6 \mathrm{bc}$ & $122,8 \mathrm{a}$ & $16,2 \mathrm{~b}$ & $76,5 \mathrm{c}$ & $104,8 \mathrm{a}$ & $117,0 \mathrm{a}$ \\
\hline Sem K & $104,2 \mathrm{c}$ & $63,0 \mathrm{bc}$ & $10,1 \mathrm{c}$ & $66,3 \mathrm{c}$ & $89,4 \mathrm{~b}$ & $102,0 \mathrm{ab}$ \\
\hline Sem Ca & $178,2 \mathrm{a}$ & $148,8 \mathrm{a}$ & $14,8 b$ & $346,5 \mathrm{a}$ & $65,8 \mathrm{bc}$ & $145,8 \mathrm{a}$ \\
\hline Sem Mg & $99,2 \mathrm{c}$ & $100,4 a b c$ & $10,2 \mathrm{c}$ & $263,0 \mathrm{~b}$ & $59,2 \mathrm{bc}$ & $111,4 \mathrm{ab}$ \\
\hline Sem S & $99,2 \mathrm{c}$ & $125,8 \mathrm{a}$ & $11,3 \mathrm{bc}$ & $56,5 \mathrm{c}$ & $63,8 b c$ & $105,6 \mathrm{ab}$ \\
\hline Tukey 5\% & 48,5 & 58,7 & 4,5 & 64,5 & 30,8 & 45,6 \\
\hline CV\% & 25,3 & 33,5 & 21,5 & 31,8 & 26,8 & 24,2 \\
\hline
\end{tabular}

Os sintorras de deficiência dos macronutrientes ( $N, P, K, \mathrm{Ca}, \mathrm{Mg} \in \mathrm{S}$ ), descritos neste trabalho, são de aspecto qualitativo e podem nāo caracterizar exatamente sintomas que eventualmente apareçam em plantas de amendoim cultivadas em condições de campo.

Nitrogênio: sua falta na planta de amendoim ocasiona menor crescimento e leve amarelecimento das folhas.

Fósforo: diminuição pouco acentuada no crescimento e desenvolvimento das plantas.

Potássio: sua falta provoca crescimento reduzido das plantas; de início, aparecem manchas castanhas nos bordos foliares, que progridem, afetando toda a folha e causando aspecto de queima necrótica, seguida da morte do tecido foliar e queda das folhas.

Cálcio: sua deficiência acarreta redução drástica no crescimento das plantas, folhas pequenas com crescimento irregular e pontos de necrose no tecido.

Magnésio: da sua deficiência resultam plantas pouco desenvolvidas e com folhas cloróticas. 


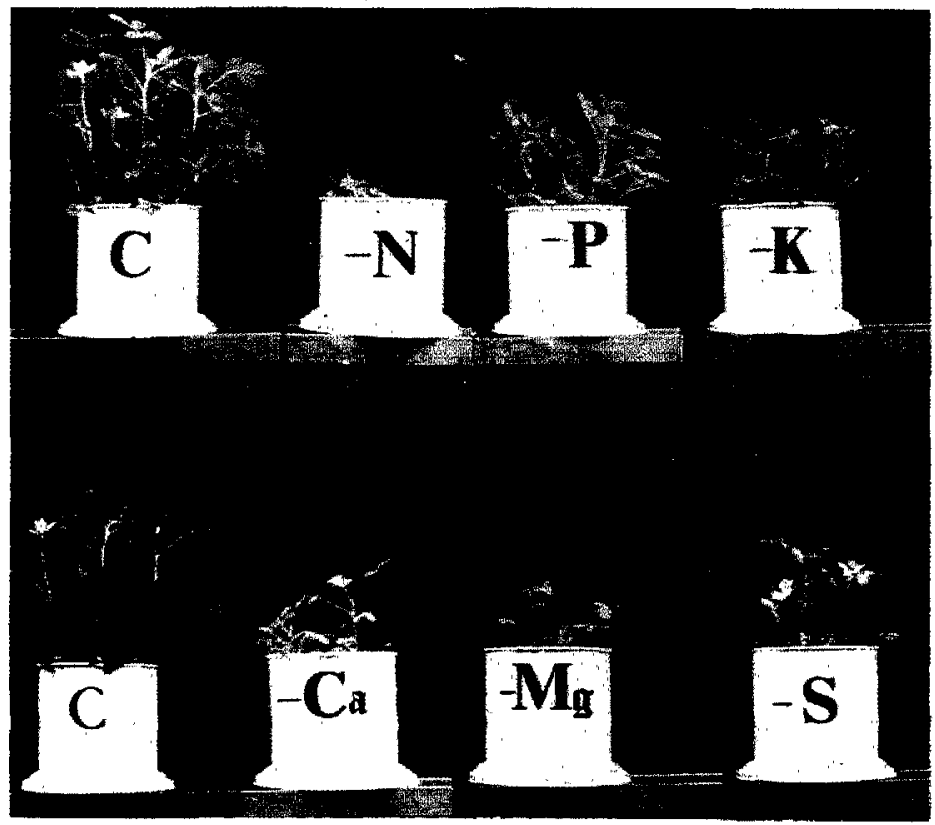

FIGURA 1. Plantas de amendoim tratadas com omissão de nitrogênio, fósforo, potássio, cálcio, magnésio e enxofre em comparação com as do tratamento completo.

Enxofre: essa falta provoca plantas pouco desenvolvidas, com folhas amarelo-pálidas, cuja nervura central apresenta coloração mais pálida, contrastando com o amarelado dos bordos.

Sintomas de deficiência de nutrientes minerais em plantas de amendoim, semelhantes aos obtidos no presente trabalho, foram descritos por HARRIS \& BLEDSOE (1951) e REID \& YORK JUNIOR (1958).

\section{CONCLUSŌES}

1. A ausência de qualquer macronutriente acarretou redução no crescimento, desenvolvimento e peso da matéria seca.

2. A omissão de $\mathrm{N}$ ocasionou menor teor de $\mathrm{Mg}$ e $\mathrm{K}$, ocorrendo o inverso na ausência de solução sem $\mathrm{Mg}$ ou $\mathrm{K}$.

3. A omissão de $\mathrm{K}$ provocou aumento nos teores de $\mathrm{N}, \mathrm{P}, \mathrm{Ca}, \mathrm{Mg}$ e $\mathrm{S}$.

4. As omissōes de macronutrientes em solução nutritiva acarretaram aumentos diferentes na absorção de $\mathrm{Fe}, \mathrm{Mn}, \mathrm{Cu}, \mathrm{Zn}, \mathrm{B}$ e $\mathrm{Al}$, nas plantas de amendoim. 
5. Apareceram sintomas tipicos de deficiência de macronutrientes, à exceção do $\mathrm{P}$, em que a deficiência se manifestou pelo menor desenvolvimento da planta.

\section{SUMMARY}

\section{DEFICIENCY OF MACRONUTRIENTS IN PEANUTS}

Peanut plants (Arachis hypogaea L.) were cultivated in washed sand, under greenhouse conditions, irrigated with a complete nutrient solution and nutrient solutions with absence of each macronutrient $(\mathrm{N}, \mathrm{P}, \mathrm{K}, \mathrm{Ca}$, $\mathrm{Mg}$ and S). Typical symptoms of macronutrient deficiencies were observed, except for phosphorus, related to the content of the element in the plant. The absence of macronutrients also influenced the plant dry matter production, development and height.

Index terms: peanuts, macronutrient deficiencies.

\section{REFERÊNCIAS BIBLIOGRÁFICAS}

BATAGLIA, O.C.; FURLANI, A.M.C.; TEIXEIRA, J.P.F.; FURLANI, P.R. \& GALLO, J.R. Métodos de análise química de plantas. Campinas, Instituto Agronômico, 1983. 48p. (Boletim Técnico, 78)

HARRIS, H.C. \& BLEDSOE, R.W. The Peanut: the unpredictable legume. Washington, D.C., The National Fertilizer Association, 1951. chap. 4, p.89-121.

HOAGLAND, D.R. \& ARNON, D.I. The water culture method for growing plants without soil. Berkeley, California Agricultural Experiment Station, 1950. 32p. (Boletim, 347)

REID, P.H. \& YORK JUNIOR, E.T. Effect of nutrient deficiencies on growth and fruiting characteristics of peanuts in sand cultures. Agronomy Journal, 50:63-67, 1958.

SARRUGE, J.R. \& HAAG, P. Soluçōes nutritivas. Summa Phytopatologica, Piracicaba, 1:231-233, 1975 . 